\title{
The Mixed Origin of the Galactic Thick Disk $\dagger$
}

\section{W. Y. Cui ${ }^{1,2}$, C. Liu ${ }^{3}$, P. de Laverny ${ }^{4}$, A. Recio-Blanco ${ }^{4}$ and G. Van de Ven ${ }^{5}$}

${ }^{1}$ Department of Physics, Shandong University, Jinan, Shandong 250100, China email: wycui@bao.ac.cn

${ }^{2}$ Department of Physics, Hebei Normal University, Shijiazhuang, Hebei 050024, China

${ }^{3}$ National Astronomical observatories, Chinese Academy of Sciences, Beijing 100012, China

${ }^{4}$ Observatoire de la Côte d'Azur, Dpt. Cassiopée UMR6 202, 06304 Nice Cedex 4, France

${ }^{5}$ Max-Planck-Institut für Astronomie, Königstuhl 17, D-69117 Heidelberg, Germany

Abstract. We found that the thick disk stars of the Galaxy should form from gas-rich mergers and minor merger or radial migration.

Keywords. Galaxy, abundances, disc, formation, kinematics and dynamics.

\section{Introduction}

Since the thick disk of the Milky Way has been discovered, many formation scenarios were proposed. However, it is still a debated issue. In this work, we investigate the chemo-orbital properties of 493 disk stars, to study the origin of the thick disk stars. The abundance data were compiled by Venn et al. (2004).

\section{Results}

We follow Liu \& van de Ven (2012) to use the circularity $L_{z} / L_{c}$ to separate the stars into two groups: the CIRC stars with $L_{z} / L_{c}>0.85$ and the ECC stars with $L_{z} / L_{c}<0.8$.
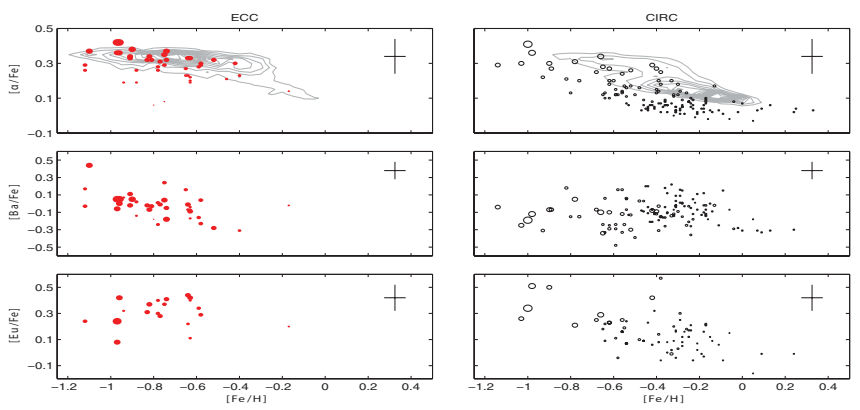

Figure 1. $[\mathrm{X} / \mathrm{Fe}]$ vs. $[\mathrm{Fe} / \mathrm{H}]$ for ECC (filled circles) and CIRC stars (unfilled circles).

Different evolution tracks of $[\mathrm{X} / \mathrm{Fe}]$ vs. $[\mathrm{Fe} / \mathrm{H}]$ imply different origins of the ECC and CIRC stars (figure 1). The ECC stars are likely originated from gas-rich mergers and the thick disk CIRC stars are likely heated from the old thin disk stars through minor merger or radial migration.

$\dagger$ This work is supported be NSFC 1100302, U1231119, 11273011, CPSF 2013M531587, NSFH A2011205102, CPRC034. 